\title{
RESURRECTION OF HANCEA AND LECTOTYPIFICATION OF ADISCA (EUPHORBIACEAE)
}

\author{
S.E.C. SIERRA, K.K.M. KULJU, J.F. VELDKAMP \& P.C. VAN WELZEN \\ Nationaal Herbarium Nederland, Universiteit Leiden branch, P.O. Box 9514, \\ 2300 RA Leiden, The Netherlands; e-mail: sierra@nhn.leidenuniv.nl
}

\begin{abstract}
SUMMARY
Based on phylogenetic studies with DNA sequence data, the formerly monotypic genus Cordemoya was recently expanded with 16 additional species previously assigned to Mallotus. However, the earliest legitimate generic name available is Hancea, and, therefore, Cordemoya is here reduced to Hancea (20 new combinations are proposed). Subsequently, the subgenus and section Diplochlamys from Asia are renamed to Hancea. Additionally, the genus Adisca is lectotypified with Adisca floribundus (= Mallotus floribundus).
\end{abstract}

Key words: Euphorbiaceae, Adisca, Cordemoya, Diplochlamys, Hancea, Mallotus, Madagascar, Malesia, Mascarenes.

\section{INTRODUCTION}

Based on a well-supported clade obtained with DNA sequence data (Kulju et al., 2007, f. 2), the genus Cordemoya Baill. (formerly monotypic with $C$. integrifolia (Willd.) Baill. from the Mascarene Islands as single species) was expanded by Sierra et al. (2006) with sixteen additional species previously assigned to Mallotus Lour. Pollen and leaf anatomical studies revealed characters that are important for the delimitation of both genera. Cordemoya can be distinguished by the presence of pollen ornamentation with scabrae and by capitate glandular hairs with multicellular stalks and sessile peltate-stellate hairs with a central cell, while Mallotus is recognized by the presence of a perforate/micro-reticulate pollen ornamentation with scabrae and by spherical to disc-shaped multicellular glandular hairs. The rank of subgenus was assigned to the two strongly supported and geographically distinct Cordemoya clades present in the phylogeny of Kulju et al. (2007): subg. Cordemoya from Madagascar and the Mascarenes and subg. Diplochlamys (Müll.Arg.) S.E.C. Sierra, Kulju \& Welzen from Asia; the latter was divided into two sections: Diplochlamys (former Mallotus sect. Hancea (Seem.) Pax \& K. Hoffm.) and Oliganthae (Airy Shaw) S.E.C. Sierra, Kulju \& Welzen. For a complete synonymy list of the species of subg. Hancea see Slik \& Welzen (2001). For subg. Hancea sect. Oliganthae and subg. Cordemoya see Sierra et al. (2006).

The epithet 'Hancea' was usually associated with use on the sectional level within Mallotus. However, its generic (and subgeneric) status must also be resurrected, as it is the earliest legitimate generic name available for the basal clade in the phylogeny by Kulju et al. (2007): Hancea Seem. was published in 1857 while Cordemoya was 
published in 1861. There is even an older name, the first generic name is Boutonia Bojer (1837), however it was invalidly described and its later validation by Bojer in 1846 created a later homonym.

Furthermore, among the species recognized in the circumscription of Cordemoya by Sierra et al. (2006), Cordemoya subpeltata (Blume) M. Aparicio has as synonym Adisca subpeltata Blume. Until now the genus Adisca Blume was not yet lectotypified; one could decide to select $C$. subpeltata as the type of Adisca, and it would become the oldest available generic name for Cordemoya. However, we opt to appoint Adisca floribunda Blume as the lectotype of Adisca, as was implicitly done by Slik \& Welzen (2001), who included Adisca in the synonymy of Mallotus sect. Stylanthus (Rchb.f. \& Zoll.) Pax \& K. Hoffm. (from the species included in Adisca by Blume, Airy Shaw (1968: 388) selected A. floribunda as the type of Mallotus sect. Stylanthus). From 1 January 2001, the International Code of Botanical Nomenclature (McNeill et al., 2006) states that one should mention "here designated or an equivalent" (Art. 7.11) and this information is lacking in the publication of Slik et al. (2001).

\section{NEW COMBINATIONS IN HANCEA}

\section{HANCEA}

Hancea Seem. (1857) 409. - Mallotus Lour. sect. Hancea (Seem.) Pax \& K. Hoffm. (1914) 199. - Type: Hancea hookeriana Seem.

Cordemoya Baill. (1861) 255. - Boutonia Bojer (1837) 282, nom. nud.; (1846) 151, non DC. (1838). - Mallotus Lour. sect. Cordemoya (Baill.) Müll.Arg. (1865) 186. - Echinus Lour. sect. Cordemoya (Baill.) Baill. (1891) 977. - Cordemoya Baill. subg. Cordemoya: S.E.C. Sierra, Kulju \& Welzen in Sierra et al. (2006) 526. - Type: Boutonia mascareinensis Bojer [= Hancea integrifolia (Willd.) S.E.C. Sierra, Kulju \& Welzen].

Diplochlamys Müll. Arg. (1864) 539; (1866) 1023. - Mallotus Lour. sect. Diplochlamys (Müll.Arg.) Baill. ex T. Durand (1888) 370; Müll. Arg. ex Pax (1890) 55, isonym. - Cordemoya Baill. subg. Diplochlamys (Müll.Arg.) S.E.C. Sierra, Kulju \& Welzen in Sierra et al. (2006) 532. - Cordemoya Baill. sect. Diplochlamys (Müll.Arg.) S.E.C. Sierra, Kulju \& Welzen in Sierra et al. (2006) 533. - Type: Diplochlamys griffithianus Müll.Arg. [= Hancea griffithiana (Müll.Arg.) S.E.C. Sierra, Kulju \& Welzen].

Deuteromallotus Pax \& K. Hoffm. (1914) 212. - Type: Deuteromallotus acuminatus (Baill.) Pax \& K. Hoffm. [= Hancea acuminata (Baill.) S.E.C. Sierra, Kulju \& Welzen].

Mallotus Lour. sect. Oliganthae Airy Shaw (1968) 389. - Cordemoya Baill. sect. Oliganthae S.E.C. Sierra, Kulju \& Welzen in Sierra et al. (2006) 535. - Type: Mallotus subpeltatus Blume [= Hancea subpeltata (Blume) S.E.C. Sierra, Kulju \& Welzen].

\section{Subgenus Cordemoya (Baill.) S.E.C. Sierra, Kulju \& Welzen, comb. nov.}

Cordemoya Baill. (1861) 255. - Boutonia Bojer (1837) 282, nom. nud.; (1846) 151, non DC. (1838). - Mallotus Lour. sect. Cordemoya (Baill.) Müll.Arg. (1865) 186. - Echinus Lour. sect. Cordemoya (Baill.) Baill. (1891) 977. - Cordemoya Baill. subg. Cordemoya: S.E.C. Sierra, Kulju \& Welzen in Sierra et al. (2006) 526. - Type: Boutonia mascareinensis Bojer [= Hancea integrifolia (Willd.) S.E.C. Sierra, Kulju \& Welzen].

Deuteromallotus Pax \& K. Hoffm. (1914) 212. - Type: Deuteromallotus acuminatus (Baill.) Pax $\&$ K. Hoffm. [= Hancea acuminata (Baill.) S.E.C. Sierra, Kulju \& Welzen]. 
1. Hancea acuminata (Baill.) S.E.C. Sierra, Kulju \& Welzen, comb. nov.

Boutonia acuminata Baill. (1858) 401. - Lectotype (McPherson, 1995): Chapelier s.n. (P), Madagascar.

2. Hancea capuronii (Léandri) S.E.C. Sierra, Kulju \& Welzen, comb. nov.

Deuteromallotus capuronii Léandri (1957, '1956') 605. - Type: SF (MAD) [Capuron] 8713 (holo P; iso MO), Madagascar, Minombo, Amboditavolo.

3. Hancea integrifolia (Willd.) S.E.C. Sierra, Kulju \& Welzen, comb. nov.

Ricinus integrifolius Willd. (1805) 567. - Lectotype (Coode, 1982): Unknown s.n. (Herb. Willd. 17934, B, here limited to the sheet with Radlkofer's notes (IDC microfiche no. 1261)), Mauritius.

4. Hancea spinulosa (McPherson) S.E.C. Sierra, Kulju \& Welzen, comb. nov.

Mallotus spinulosus McPherson (1995) 170. - Type: Schatz \& Lowry 1301 (holo MO; iso G, K, P, S, WAG), Madagascar, Tamatave, Ambila-Lemaitso.

\section{Subgenus Hancea}

\section{Section Hancea Seem.}

Hancea Seem. (1857) 409. - Mallotus Lour. sect. Hancea (Seem.) Pax \& K. Hoffm. (1914) 199. - Type: Hancea hookeriana Seem.

Diplochlamys Müll. Arg. (1864) 539; (1866) 1023. - Mallotus Lour. sect. Diplochlamys (Müll. Arg.) Baill. ex T. Durand (1888) 370; Müll.Arg. ex Pax (1890) 55, isonym. - Cordemoya Baill. subg. Cordemoya: S.E.C. Sierra, Kulju \& Welzen in Sierra et al. (2006) 532. - Cordemoya Baill. sect. Diplochlamys (Müll.Arg.) S.E.C. Sierra, Kulju \& Welzen in Sierra et al. (2006) 533. - Type: Diplochlamys griffithianus Müll.Arg. [= Hancea griffithiana (Müll.Arg.) S.E.C. Sierra, Kulju \& Welzen].

5. Hancea cordatifolia (Slik) S.E.C. Sierra, Kulju \& Welzen, comb. nov.

Mallotus cordatifolius Slik (1998) 225. - Type: PNH (Gutierrez et al.) 117545 (holo L), Philippines, Samar Island, Mt Sohoon.

6. Hancea eucausta (Airy Shaw) S.E.C. Sierra, Kulju \& Welzen, comb. nov.

Mallotus eucaustus Airy Shaw (1969) 80. - Type: S (Sibat ak Luang) 23630 (holo K; iso L), Borneo, Sarawak, Third Division, Bukit Iju, Ulu Arip, Balingian.

7. Hancea grandistipularis (Slik) S.E.C. Sierra, Kulju \& Welzen, comb. nov.

Mallotus grandistipularis Slik (1998) 227. - Type: Burley, Tukirin et al. 2021 (holo L; iso CANB, $\mathrm{K}, \mathrm{US}$ ), Indonesia, Sumatra, Riau Province, Tigapulu, $5 \mathrm{~km} \mathrm{~W}$ of Talanglakat.

8. Hancea griffithiana (Müll.Arg.) S.E.C. Sierra, Kulju \& Welzen, comb. nov. Diplochlamys griffithianus Müll. Arg. (1864) 539. - Type: Griffith KD 4961 (holo K). 
9. Hancea hirsuta (Elmer) S.E.C. Sierra, Kulju \& Welzen, comb. nov.

Mallotus hirsutus Elmer (1915) 2648. - Type: Elmer 13480 (holo PNH?; iso K, L, NY), Philippines, Mindanao, Agusan Province, Cabadbaran (Mt Urdaneta).

\section{Hancea hookeriana Seem.}

Hancea hookeriana Seem. (1857) 409. - Type: Champion s.n. (holo K), Hong Kong.

11. Hancea kingii (Hook.f.) S.E.C. Sierra, Kulju \& Welzen, comb. nov.

Mallotus kingii Hook.f. (1887) 439. - Type: King's collector 7414 (holo K; iso G, SING), Malaya, Peak.

12. Hancea longistyla (Merr.) S.E.C. Sierra, Kulju \& Welzen, comb. nov.

Mallotus longistylus Merr. (1920) 560. - Type: BS (Ramos \& Pascasio) 35173 (holo PNH†; iso K), Philippines.

13. Hancea papuana (J.J. Sm.) S.E.C. Sierra, Kulju \& Welzen, comb. nov.

Mallotus hookerianus (Seem.) Müll.Arg. var. papuanus J.J. Sm. (1912) 787, t. 137. - Type: Gjellerup 316 (holo L; iso A, BO, K, P, U), Irian Jaya, Bivak Hollandia.

14. Hancea penangensis (Müll.Arg.) S.E.C. Sierra, Kulju \& Welzen, comb. nov.

Mallotus penangensis Müll. Arg. (1865) 186. - Type: Wallich 8576 (holo G; iso A, K, P), Malaysia, Pulau Penang.

15. Hancea stipularis (Meijer ex Airy Shaw) S.E.C. Sierra, Kulju \& Welzen, comb. nov.

Mallotus stipularis Meijer ex Airy Shaw (1968) 398. - Type: Lörzing 14746 (holo K; iso L), Sumatra, N Sibajak, W of Bandarbaru.

16. Hancea wenzeliana (Slik) S.E.C. Sierra, Kulju \& Welzen, comb. nov.

Mallotus wenzelianus Slik (1998) 229. - Type: Wenzel 2697 (holo G; iso A, UC), Philippines, Mindanao, Surigao Province, Jubud.

Section Oliganthae (Airy Shaw) S.E.C. Sierra, Kulju \& Welzen, comb. nov.

Mallotus Lour. sect. Oliganthae Airy Shaw (1968) 389. - Cordemoya Baill. sect. Oliganthae S.E.C. Sierra, Kulju \& Welzen in Sierra et al. (2006) 535. - Type: Mallotus subpeltatus Blume [= Hancea subpeltata (Blume) S.E.C. Sierra, Kulju \& Welzen].

17. Hancea subpeltata (Blume) M. Aparicio, comb. nov.

Adisca subpeltata Blume (1826) 610. - Lectotype (Sierra et al., 2006): Blume 1510 (holo L), Java, Krawong. 


\section{LECTOTYPIFICATION OF ADISCA}

Adisca Blume (1826) 609; Slik \& Welzen (2001) 31. - Lectotype (selected here): Adisca floribunda Blume [= Mallotus floribundus (Blume) Müll. Arg.].

\section{ACKNOWLEDGEMENTS}

Special thanks are due to D.J. Mabberley for opening our eyes and to the comments of the anonymous reviewer.

\section{REFERENCES}

Airy Shaw, H.K. 1968. Malesian and other Asiatic Euphorbiaceae. Kew Bull. 21: 379-400.

Airy Shaw, H.K. 1969. Malesian and other Asiatic Euphorbiaceae. Kew Bull. 23: 80-82.

Baillon, H.E. 1858. Étude générale du groupe des Euphorbiacées. Masson, Paris.

Baillon, H.E. 1861. Species Euphorbiacearum. Euphorbiacées africaines. Adansonia 1: 255-256.

Baillon, H.E. 1891. Liste des plantes de Madagascar. Bull. Mens. Soc. Linn. Paris 2: 977-978.

Blume, C.L. 1826. Adisca. In: Bijdragen tot de Flora van Nederlandsch-Indië 11: 609-611. Lands Drukkerij, Batavia.

Bojer, W. 1837. Hortus Mauritianus. Aime Mararot \& Co., Mauritius.

Bojer, W. 1846. Boutonia. In: L. Bouton, Trav. Soc. Hist. Nat. Maurice 1842-1846: 151-153.

Coode, M.J.E. 1982. Euphorbiaceae. In: Flora des Mascareignes 160: 50-51. The Sugar Industries Research Institute, Mauritius. L'Office de la Recherche Scientifique et Technique Outre-mer, Paris; The Royal Botanical Gardens, Kew.

De Candolle, A.P. 1838. Revue sommaire de la famille des Bignoniacées. Biblioth. Universelle Genève II, 17: 134, reprinted in Ann. Sci. Nat., Bot. II, 11 (1839): 296.

Durand, T.A. 1888. Index generum phanerogamorum: 370. Durand, Brussel.

Elmer, A.D.E. 1915. Two hundred twenty six new species. Leafl. Philipp. Bot. 7: 2648.

Hooker, J.D. 1887. Flora of British India 5. Reeve \& Co., London.

Kulju, K.K.M., S.E.C. Sierra, S.G.A. Draisma, R. Samuel \& P.C. van Welzen. 2007. Molecular phylogeny of Macaranga, Mallotus, and related genera (Euphorbiaceae s.s.): insights from plastid and nuclear DNA sequence data. Amer. J. Bot. 94: 1726-1743.

Léandri, J. 1957 ('1956’). Euphorbiacee malgaches nouvelles recoltées par M.R Capuron. Bull. Soc. Bot. France 103: 605-606.

McNeill, J., F.R. Barrie, H.M. Burdet, V. Demoulin, D.L. Hawksworth, K. Marhold, D.H. Nicolson, J. Prado, P.C. Silva, J.E. Skog, J.H. Wiersema \& N.J. Turland (eds.). 2006. International Code of Botanical Nomenclature (Vienna Code) adopted by the Seventeenth International Botanical Congress Vienna, Austria, July 2005. Regnum Veg. 146.

McPherson, G.D. 1995. On Mallotus and Deuteromallotus (Euphorbiaceae) in Madagascar. Bull. Mus. Natl. Hist. Nat., B, Adansonia IV, 17: 170.

Merrill, E.D. 1920. Notes on Philippine Euphorbiaceae, III. Philipp. J. Sci. 16: 559-561.

Müller Argoviensis, J. 1864. Neue Euphorbiaceen des Herbarium Hooker in Kew. Flora 47: 539.

Müller Argoviensis, J. 1865. Euphorbiaceae. Linnaea 34: 184-197.

Müller Argoviensis, J. 1866. Euphorbiaceae tribus Acalypheae. In: A. de Candolle (ed.), Prodr. 15, 2: 956-958, 1023. Masson \& Filii, Paris.

Pax, F. A. 1890. Euphorbiaceae. In: A. Engler, Die natürlichen Pflanzenfamilien 3, 5: 53-55. Engelmann, Leipzig.

Pax, F.A. \& K. Hoffmann. 1914. Euphorbiaceae-Acalyphae-Mercurialinae. In: A. Engler (ed.), Das Pflanzenreich IV.147.vii: 145-212, 394-397. Engelmann, Leipzig.

Seemann, B.C. 1857. Flora of the Island of Hong Kong. The Botany of the Voyage of H.M.S. Herald: 409-410. Reeve \& Co., London. 
Sierra, S.E.C., M. Aparicio, K.K. M. Kulju,Ž. Fišer, P.C. van Welzen \& R.W.J.M. van der Ham. 2006. Re-shaping Mallotus [part 1]: Expanded circumscription and revision of the genus Cordemoya (Euphorbiaceae). Blumea 51: 519-540.

Slik, J.W.F. 1998. Three new Malesian species of Mallotus section Hancea (Euphorbiaceae). Blumea 43: $225-232$.

Slik, J.W.F. \& P.C. van Welzen. 2001. A taxonomic revision of Mallotus sections Hancea and Stylanthus (Euphorbiaceae). Blumea 46: 3-66.

Smith, J.J. 1912. Euphorbiaceae. Nova Guinea 8, 4: 779-796, t. 130-142.

Willdenow, C.L. 1805. Species Plantarum, ed. 4, 1. Nauk, Berlin. 